\title{
ESTUDO POR ESPECTROMETRIA DE MASSAS DE SOLUÇÕES DE HIDRÓXI(TOSILÓXI)IODOBENZENO: PROPOSTAS PARA MECANISMOS DE DESPROPORÇÃO
}

\author{
Ramon S. Vasconcelos e Luiz F. Silva Jr* \\ Departamento de Química Fundamental, Instituto de Química, Universidade de São Paulo, CP 26077, 05513-970 São Paulo - SP, \\ Brasil
}

\section{Norberto P. Lopes}

Departamento de Física e Química, Faculdade de Ciências Farmacêuticas de Ribeirão Preto, Universidade de São Paulo, Av. do Café, s/n, 14040-903 Ribeirão Preto - SP / Departamento de Química Fundamental, Instituto de Química, Universidade de São Paulo, CP 26077, 05513-970 São Paulo - SP, Brasil

Recebido em 24/1/12; aceito em 23/4/12; publicado na web em 27/7/12

\begin{abstract}
STUDY BY MASS SPECTROMETRY OF SOLUTIONS OF [HYDROXY(TOSYLOXY)IODO]BENZENE: PROPOSED DISPROPORTIONATION MECHANISMS. Solutions of [hydroxy(tosyloxy)iodo]benzene (HTIB or Koser's reagent) in acetonitrile were analyzed using high resolution electrospray ionization mass spectrometry (ESI-MS) and electrospray ionization tandem mass spectrometry (ESI-MS/MS) under different conditions. Several species were characterized in these analyses. Based on these data, mechanisms were proposed for the disproportionation of the iodine(III) compounds in iodine(V) and iodine(I) species.
\end{abstract}

Keywords: hypervalent iodine; electrospray ionization mass spectrometry; disproportionation.

\section{INTRODUÇÃO}

Reagentes de iodo hipervalente são atualmente uma ferramenta essencial em química orgânica sintética, pois promovem uma série de transformações de maneira eficiente, ${ }^{1-11}$ incluindo um enorme número de aplicações na síntese total de produtos naturais. ${ }^{8} \mathrm{O}$ crescente uso dos reagentes de iodo hipervalente está relacionado com o fato que representam em muitas situações uma substituição a metais de transição tóxicos. ${ }^{12}$ Além disso, a utilização de reagentes de iodo hipervalente em substituição a metais de transição evita a presença de traços de metais nos produtos finais, que é um problema recorrente na indústria farmacêutica. ${ }^{13}$ Os compostos de iodo hipervalente mais conhecidos estão divididos em duas classes. A primeira, compreende os de estado de oxidação (III) ${ }^{13-15}$ representados pelo iodobenzeno diacetato $\left(\mathrm{PhI}(\mathrm{OAc})_{2}\right.$, DIB), o hidróxi(tosilóxi)iodobenzeno $(\mathrm{PhI}(\mathrm{OTs}) \mathrm{OH}$, $\mathrm{HTIB})$, o iodobenzeno bis-trifluoro-acetato $\left(\mathrm{PhI}\left(\mathrm{CF}_{3} \mathrm{CO}_{2}\right)_{2}\right.$, PIFA) e sais de iodônio. Na segunda, estão incluídos os de estado de oxidação (V), ${ }^{7,16,17}$ como o ácido 2-iodóxibenzoico (IBX) e a periodinana de Dess-Martin (DMP) (Figura 1).

Soluções de iodo(III) espontaneamente iniciam uma reação de desproporção, gerando espécies de iodo(I) e de iodo(V). ${ }^{19,20}$ Essa reação de desproporção foi utilizada na oxidação de alcoóis, utilizando DIB em acetonitrila aquosa na presença de $\mathrm{RuCl}_{3}$ catalítico. Os autores mostraram que a adição de $\mathrm{RuCl}_{3}$ provoca uma desproporção quase instantânea do DIB a $\mathrm{PhIO}_{2}$ e PhI, sendo que o primeiro promove a oxidação do álcool. Isso foi constatado ao isolarem um sólido branco formado logo após a adição do catalisador, cujo espectro de RMN e ponto de fusão concordam com a formação do reagente de iodo(V) $\mathrm{PhIO}_{2}$. O mesmo grupo utilizou estratégia análoga em outras aplicações, ${ }^{21}$ incluindo a preparação e aplicação de iodilarenos. ${ }^{22-24} \mathrm{~A}$ desproporção de compostos de I(III) também foi investigada por Kita e colaboradores que, após realizarem a oxidação de alcoóis utilizando $\mathrm{PhIO} / \mathrm{KBr}$ em água, ${ }^{25}$ fizeram um estudo sobre o mecanismo da reação para averiguar o envolvimento de espécies de I(V), o que não se confirmou. ${ }^{26}$ Apesar da importância da desproporção de reagentes

*e-mail: luizfsjr@iq.usp.br
Reagentes de lodo Hipervalente(III):

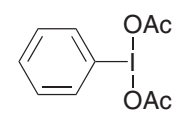

(Diacetóxi)iodobenzeno DIB, PIDA, BAIB

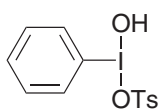

[Hidróxi(tosilóxi)iodo]benzeno HTIB, HTI, Reagente de Koser

Reagentes de lodo Hipervalente(V):

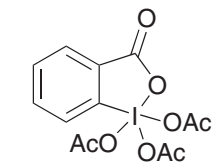

Periodinana de Dess-Martin DMP

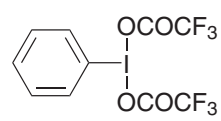

[Bis(trifluoroacetóxi)]iodobenzeno BTI, PIFA

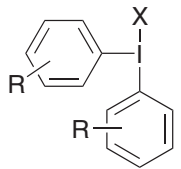

Diaril- $\lambda^{3}$-iodana ou Sal de Diariliodônio

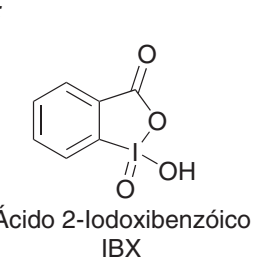

Figura 1. Reagentes de iodo hipervalente amplamente utilizados e seus acrônimos $^{18}$

de I(III), pouco se tem feito para entender o mecanismo desta reação. Richter, Koser e colaboradores fizeram um estudo das espécies de iodo existentes em soluções aquosas de HTIB e de PhI(OMs)OH (hidróxi(mesilóxi)iodobenzeno, HMIB) por espectroscopia de UV-Vis e potenciometria. ${ }^{27}$ Segundo os autores, a dissolução do HTIB e do HMIB não se resume a uma simples solvatação do reagente, envolvendo reações mais complexas. Foi proposto que a espécie dimérica $\mathrm{PhI}(\mathrm{OH}) \mathrm{OI}\left(\mathrm{OH}_{2}\right) \mathrm{Ph}^{+}$desproporciona termicamente, gerando $\mathrm{PhI}$ e $\mathrm{PhIO}_{2}$. Esta reação redox, na qual duas espécies de iodo(III) levam a compostos de iodo(I) e iodo(V), foi primeiramente mencionada por Willgerodt, em $1892 .{ }^{28}$ A presença de dímeros como 1-4 também foi constatada por nosso grupo, ${ }^{29}$ enquanto que Zhdankin e colaboradores relataram a ocorrência do trímero 5 (Figura 2). ${ }^{30}$

Uma maneira para caracterizar compostos presentes em uma solução é a técnica de espectrometria de massas com ionização por 


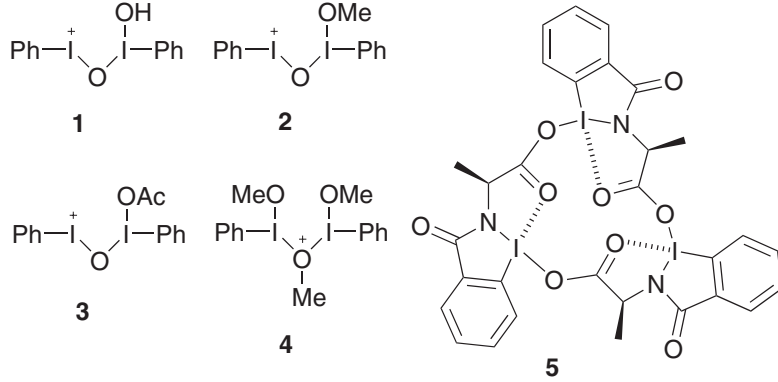

Figura 2. Estruturas de dímeros e de um trímero contendo iodo(III)

eletrospray (IES-EM) que é capaz de detectar e caracterizar intermediários reacionais sem a necessidade de isolamento, mesmo em baixas concentrações. ${ }^{31-35}$ Não por acaso muitos grupos têm usado IES-EM para estudo de compostos de iodo hipervalente. ${ }^{24,26,29,36-39}$ Sam e colaboradores conseguiram identificar as espécies de iodo $[\mathrm{PhIOH}]^{+}$, $[\mathrm{PhIOMe}]^{+}$e o dímero 4 em uma solução de bleomicina e PhIO em $\mathrm{MeOH}$ empregando IES-EM. ${ }^{36} \mathrm{Li}$ e colaboradores ${ }^{37}$ fizeram um estudo mecanístico da resolução cinética de alcoóis secundários promovida por DIB catalisada por complexos de [MnIII(salen)] na presença de $\mathrm{KBr}$ em água/diclorometano na proporção de 2:1. Ao adicionar apenas o catalisador juntamente com o DIB no solvente, o espectro de IESEM revelou como espécie principal o íon [MnIII(salen)-PhI(OAc) $]_{2}^{+}$ relativo ao DIB coordenado ao complexo de Mn(III). Em seguida, foi adicionado o álcool benzílico, constatando-se o desaparecimento do pico principal e o surgimento de um sinal que foi atribuído à espécie $\left[\mathrm{PhIOMn}\right.$ (salen) $\left[\mathrm{OCH}\left(\mathrm{CH}_{3}\right) \mathrm{Ph}\right]^{+}$.

Recentemente, realizamos um estudo detalhado das propriedades em solução do DIB utilizando IES-EM, no qual foi proposto um mecanismo para a desproporção de espécies de iodo(III) em iodo(I) e $\operatorname{iodo}(\mathrm{V}) .^{40}$ Apresentamos neste artigo um trabalho análogo com outro importante reagente de iodo(III) hipervalente HTIB (ou Reagente de Koser), ${ }^{14,41}$ procurando analisar as diferenças e semelhanças com relação ao DIB.

\section{PARTE EXPERIMENTAL}

HTIB está disponível comercialmente e foi utilizado como recebido. A acetonitrila foi escolhida como solvente, pois as reações de desproporção são suficientemente lentas neste meio a ponto de ser possível acompanhar a evolução gradual da formação dos intermediários por IES-EM. Isto ocorre já que acetonitrila solvata os íons de maneira menos eficiente que em água, onde a velocidade de desproporção é mais pronunciada. Como o HTIB apresenta grupos (OH, OTs), que são facilmente protonados e/ou coordenam-se com cátions metálicos, e o $\mathrm{pH}$ dessas soluções é baixo $(\mathrm{pH}=4-5)$ as análises foram feitas em IES-EM de alta resolução no modo positivo. Todos os espectros de massas foram obtidos utilizando o espectrômetro de massas UltrOTOF Q (Bruker Daltonics, Billerica, MA, USA) equipado com uma interface ionspray. As amostras foram introduzidas utilizando seringas Hamilton (Hamilton Co., Reno, NE). Para confirmar a estrutura proposta, cada um dos íons encontrados foi selecionado e caracterizado por IES-EM/EM, usando dissociação induzida por colisão, utilizando nitrogênio como gás de colisão.

\section{RESULTADOS E DISCUSSÃO}

\section{IES-EM de uma solução recém-preparada de HTIB a $25{ }^{\circ} \mathrm{C}$}

Em uma solução recém-preparada de HTIB (até 10 min) em acetonitrila com concentração de $3,8 \mathrm{mmol} \mathrm{L}^{-1}(1,5 \mathrm{mg} / \mathrm{mL})$ e $\mathrm{pH}$ 4 , foram detectados os seguintes íons: a) cátion radical $[\mathrm{PhI}]^{++}$(obs. $\mathrm{m} / \mathrm{z}$ 203,9452, calc. 203,9436, 7,8 ppm); b) iodosilbenzeno protonado $[\mathrm{PhIOH}]^{+}$(obs. $m / z$ 220,9463, calc. 220,9463, 0,0 ppm); c) espécie de $\operatorname{iodo}(\mathrm{V})[\mathrm{PhI}(\mathrm{O}) \mathrm{OH}]^{+}$(obs. $\mathrm{m} / z, 236,9400$, calc. 236,9413, -5,5 ppm); d) $[\mathrm{PhIOTs}]^{+}$(obs. $\mathrm{m} / \mathrm{z}, 374,9504$, calc. 374,9552, -12,8 ppm); e) íon (2-iodofenil)-fenil-iodônio [(PhI)IPh] ${ }^{+} \mathbf{6}$ (obs. $\mathrm{m} / \mathrm{z}$ 406,8742, calc. 406,8794, -12,8 ppm); f) dímero 1 (obs. $m / z$ 440,8775, calc. 440,8849, $-16,8$ ppm) e g) dímero [PhIO(OTs)IPh $]^{+} 7$ (obs. $\mathrm{m} / z$ 594,8845, calc. 594,8937, -15,5 ppm) (Figura 3).

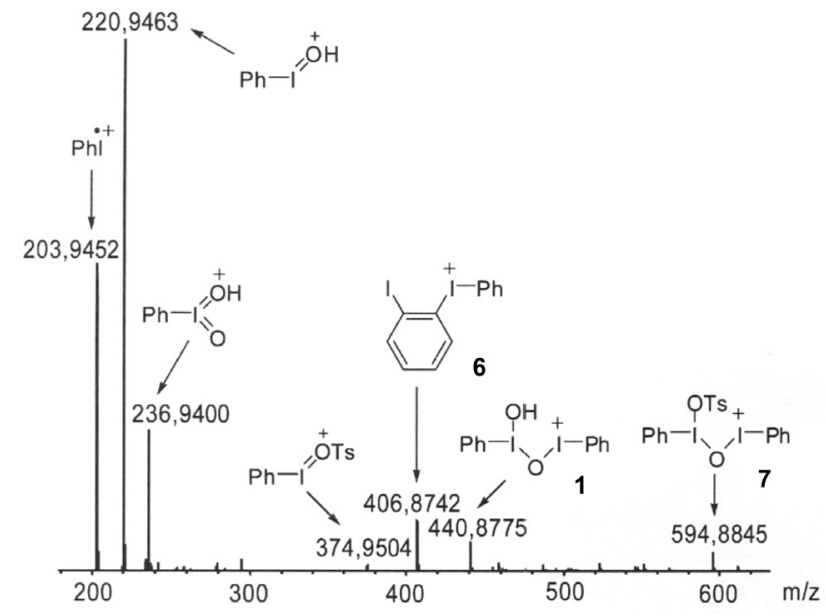

Figura 3. Espectro de massas de uma solução de HTIB em acetonitrila recém-preparada

$\mathrm{O}$ íon $[\mathrm{PhIOH}]^{+}$deve ser proveniente da protonação do grupo tosila do HTIB, seguido da saída de uma molécula neutra de ácido $p$-toluenossulfônico. Já o cátion radical $[\mathrm{PhI}]^{*+}$ deve ser gerado pela cisão homolítica da ligação I-OH do íon $[\mathrm{PhIOH}]^{+}$(Esquema 1). Alternativamente, pode ter sido gerado pela oxidação do iodobenzeno na fonte do espectrômetro (Esquema 2), como ocorre com várias outras espécies. ${ }^{42}$

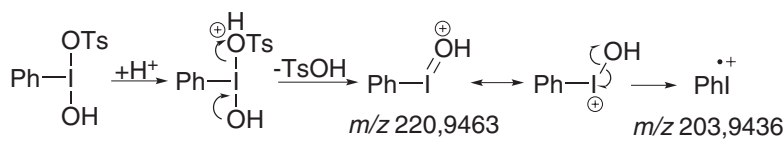

Esquema 1

$\mathrm{Phl} \underset{\text { Esquema } 2}{\stackrel{\substack{\text { oxidação na } \\ \text { fonte }}}{\underset{\text { Esql }}{ }}} \mathrm{Ph}$

$\mathrm{O}$ íon $[\mathrm{PhIOTs}]^{+}$é, possivelmente, gerado pela saída de água do HTIB protonado. Este íon pode também contribuir na formação de $[\mathrm{PhI}]^{\cdot+}$ pela cisão homolítica da ligação I-OTs (Esquema 3). A intensidade do pico do íon [PhIOTs] $]^{+}$é menor que do íon $[\mathrm{PhIOH}]^{+}$, o que não surpreende, uma vez que o ácido $p$-toluenossulfônico é um grupo de saída melhor que a água.

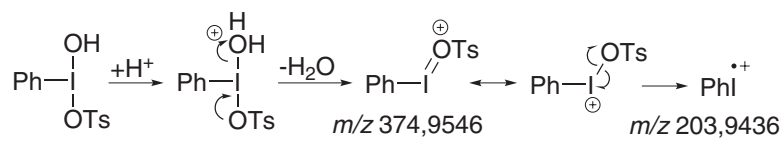

Esquema 3

O dímero 1 é análogo às espécies diméricas descritas por Richter e colaboradores ${ }^{27}$ e é o produto da adição reversível do iodosilbenzeno ao cátion $[\mathrm{PhIOH}]^{+}$(Esquema 4$)$. Masson e colaboradores ${ }^{43}$ propuseram que o dímero 1 pode desproporcionar nas espécies de I(V) e I(I), 
o que explica a formação do cátion $[\mathrm{PhI}(\mathrm{O}) \mathrm{OH}]^{+}$. Analogamente, o dímero 7 deve ser originado pela dimerização do íon [PhIOTs] $]^{+}$com PhIO (Esquema 5).

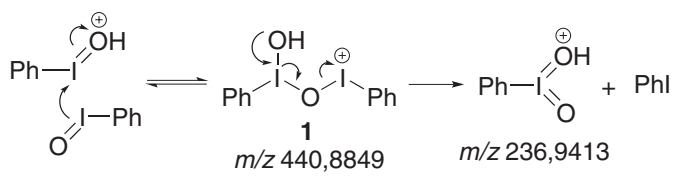

Esquema 4

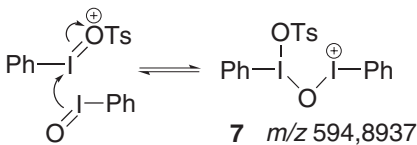

Esquema 5

Diferentemente do DIB, a ausência dos produtos cationizados com metais ou mesmo a forma protonada do HTIB pode indicar que o reagente se encontra totalmente ionizado em solução, como relatado anteriormente por Richter e colaboradores. ${ }^{27} \mathrm{~A}$ presença de espécies diméricas e de iodo(V) na solução fresca de HTIB pode ser explicada pela maior reatividade desse reagente em relação ao DIB. Esta diferença de reatividade pode ser atribuída ao fato que no HTIB a ligação I-OTs é praticamente iônica, enquanto que no DIB a ligação I-OAc é covalente. ${ }^{2,44}$ Isto faz com que o átomo de iodo(III) no HTIB tenha um caráter muito mais eletrofílico do que no DIB.

\section{IES-EM de uma solução de HTIB a $25^{\circ} \mathrm{C}$ após 24 h}

Após a identificação das espécies de iodo presentes numa solução de HTIB em acetonitrila recém-preparada, esta foi deixada em repouso à temperatura ambiente por $24 \mathrm{~h}$ e, em seguida, foi submetida a experimentos de IES-EM de alta resolução (Figura 4), de modo análogo ao realizado anteriormente para a solução de DIB..$^{40}$

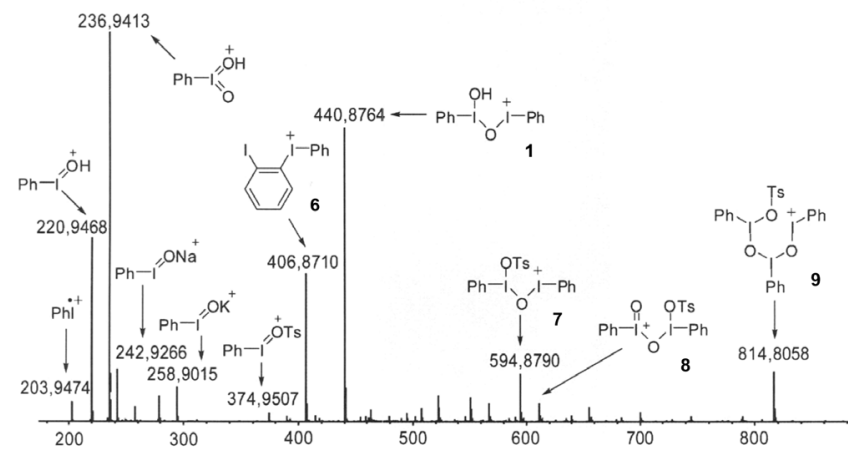

Figura 4. Espectro de massas de uma solução de HTIB em acetonitrila após $24 \mathrm{~h}$

O espectro da solução "envelhecida" de HTIB apresenta os mesmos picos que na solução fresca, contudo em proporções diferentes. Nota-se um aumento substancial das espécies 1 (obs. $m / z, 440,8764$, calc. 440,8849, -19,3 ppm), 6 (obs. $m / z$ 406,8710, calc. 406,8794, -20,6 ppm), 7 (obs. $\mathrm{m} / \mathrm{z}, 594,8790$, calc. 594,8937, -24,7 ppm) e $[\mathrm{PhI}(\mathrm{O}) \mathrm{OH}]^{+}$(obs. $\mathrm{m} / z, 236,9413$, calc. 236,9413, 0,0 ppm). Além disso, ocorreu o aparecimento dos produtos cationizados com sódio [PhIONa] $]^{+}$(obs. $\mathrm{m} / z, 242,9266$, calc. 242,9277, -4,6 ppm) e com potássio $[\mathrm{PhIOK}]^{+}$(obs. $\mathrm{m} / \mathrm{z}, 258,9015$, calc. 258,9017, $-0,77$ ppm) do iodosilbenzeno, de maneira análoga ao observado para a solução de DIB..$^{40}$ Estes íons devem ser formados a partir da coordenação dos metais alcalinos ao PhIO presente na solução de HTIB (Esquema 6).

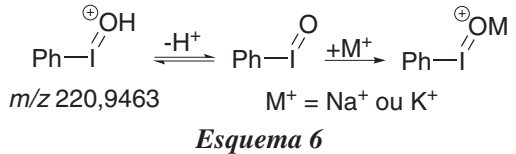

O dímero $[\mathrm{PhI}(\mathrm{OTs}) \mathrm{OI}(\mathrm{O}) \mathrm{Ph}]^{+} \mathbf{8}(\mathrm{m} / z$ estimado 610,8881$)$ possui os átomos de iodo nos estados de oxidação (III) e (V). Esta espécie deve ser gerada em solução pela adição irreversível de [PhI(O)OTs] ${ }^{+}$ ao PhIO. A migração do grupo tosila de 8 leva à formação de 11, passando pelo intermediário cíclico $\mathbf{1 0}$ (Esquema 7) ${ }^{40}$ Adicionalmente, as espécies isoméricas $\mathbf{8 , 1 0}$ e $\mathbf{1 1}$ devem se encontrar em equilíbrio e não são distinguíveis por IES-EM. Outra via para a formação de $\mathbf{1 1}$ e, por conseguinte, para as espécies $\mathbf{8}$ e $\mathbf{1 0}$ seria pela desproporção do trímero 9. Para efeito de representação nos espectros será adotada arbitrariamente a espécie $\mathbf{8}$ (Esquema 8).

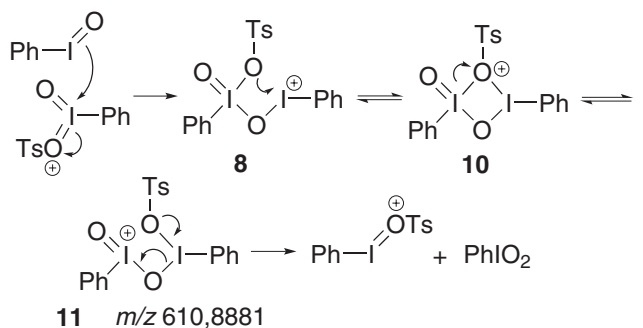

Esquema 7

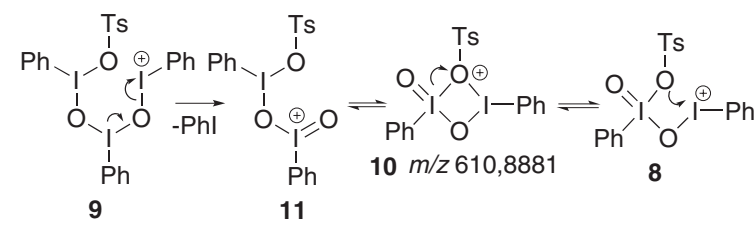

Esquema 8

Os íons 7, 8 e 9 foram selecionados para experimentos de IESEM/EM, mas sem induzir nenhuma dissociação por colisão, ou seja, fechando-se a entrada e desligando a energia do gás de colisão. Nesta condição, verificou-se que a espécie 7 (obs. $\mathrm{m} / \mathrm{z}$ 594,8917, calc. 594,8937, -3,3 ppm) (Figura 5) levou ao íon [PhIOTs] ${ }^{+}$(obs. $m / z$ 374,9528, calc. 374,9552, -6,4 ppm), evidenciando a formação reversível deste dímero. No mesmo espectro foi detectada a presença do íon iodilbenzeno tosilado $[\mathrm{PhI}(\mathrm{O}) \mathrm{OTs}]^{+}$(obs. $\mathrm{m} / \mathrm{z}$ 390,9512, calc. 390,9501, 2,8 ppm), demonstrando que a espécie de I(V) se origina do dímero 7 por um processo de desproporção espontânea em fase gasosa.

O espectro de IES-EM/EM do dímero 8 apresenta os íons $[\mathrm{PhIOH}]^{+}\left(\mathrm{m} / z\right.$ estimado 220,9463), $[\mathrm{PhI}(\mathrm{O}) \mathrm{OH}]^{+}$(obs. $\mathrm{m} / z$ 236,9441,

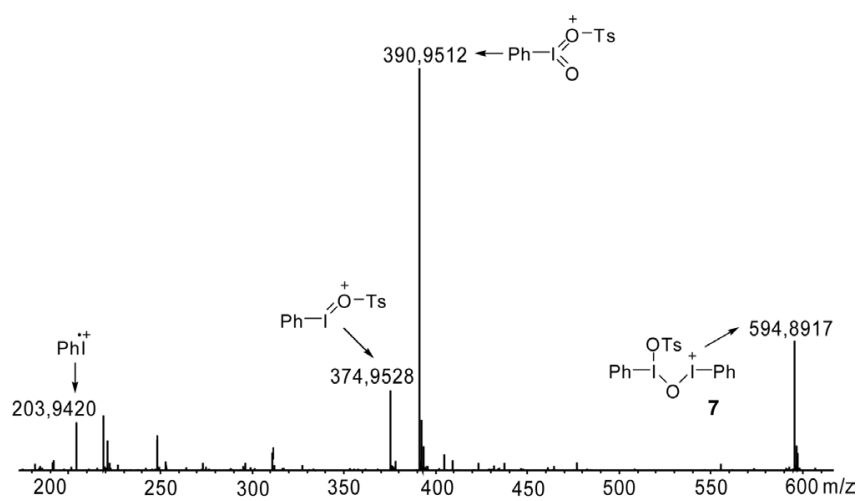

Figura 5. Espectro de IES-EM/EM do dímero 7 (energia de colisão zero) 


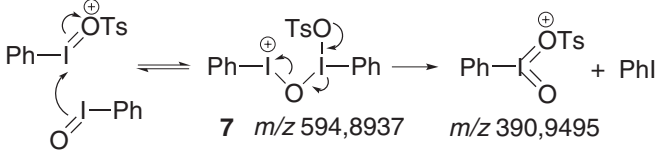

Esquema 9

calc. $236,9413,11,8$ ppm), $[\text { PhIOTs }]^{+}$(obs. $m / z$ 374,9584, calc. $374,9552,8,5$ ppm) e o dímero 1 (obs. $m / z, 440,8849$, calc. 440,8849, $0,0 \mathrm{ppm}$ ) (Figura 6).

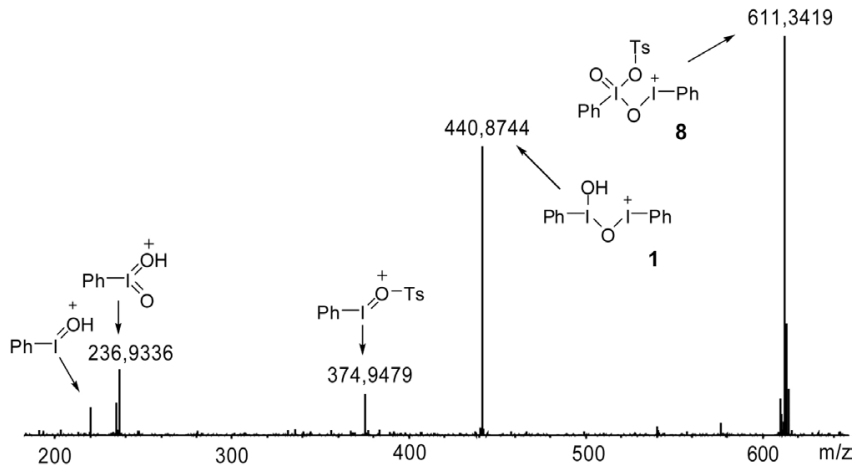

Figura 6. Espectro de IES-EM/EM do dímero 8 (energia de colisão zero)

O dímero 1 é formado pela adição eletrofílica do oxigênio ligado ao átomo de $\mathrm{I}(\mathrm{V})$ ao anel aromático. Em seguida, ocorre a rearomatização do grupo toluila pela saída do próton, que se liga ao oxigênio vizinho aos dois átomos de iodo. Por fim, acontece a clivagem do anel com a formação do instável sulfeno $\mathbf{1 2},{ }^{45,46}$ o qual gera o éster do ácido 2-hidróxi-4-metilbenzenossulfônico 13 (Esquema 10). ${ }^{47}$

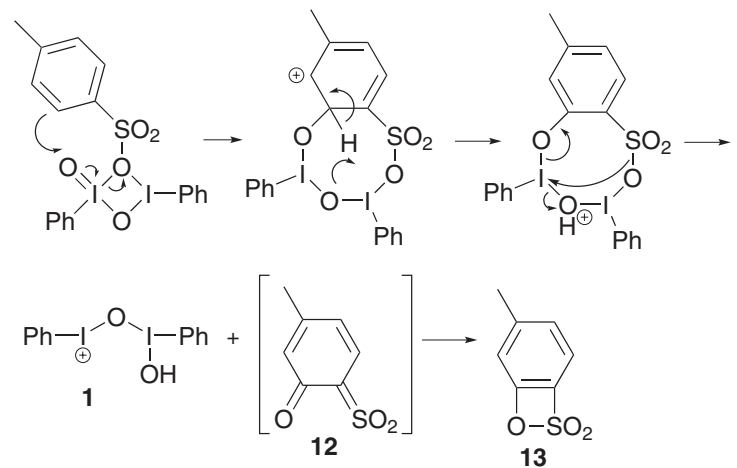

Esquema 10

$\mathrm{O}$ íon $[\mathrm{PhIOTs}]^{+}$deve ser formado provavelmente pela migração do grupo tosila de 8 formando 11, que fragmenta em [PhIOTs] ${ }^{+} \mathrm{e}$ $\mathrm{PhIO}_{2}$ (Esquema 7). O íon $[\mathrm{PhI}(\mathrm{O}) \mathrm{OH}]^{+}$(obs. $\mathrm{m} / z$ 236,9441, calc. 236,9407, 11,8 ppm) é possivelmente oriundo de uma adição eletrofílica do iodo(III) ao anel toluila, formando 14. A desprotonação assistida pelo oxigênio leva à rearomatização da espécie, gerando 15, cuja clivagem leva à liberação do íon $[\mathrm{PhI}(\mathrm{O}) \mathrm{OH}]^{+}$e formação do iodônio 16. A espécie de dupla carga 16 pode estar em equilíbrio com a forma neutra 17 (Esquema 11). O trímero [PhI(OTs)OI(Ph) $\mathrm{OIPh}]^{+} \mathbf{9}(\mathrm{m} / z$ estimado 814,8322$)$ pode ser formado pela reação do iodosilbenzeno com o dímero 7 (Esquema 12).

O iodônio 6 (obs. $m / z$ 406,8742, calc. 406,8794, -12,8 ppm) deve ser gerado a partir do trímero 9 pelo ataque eletrofílico do grupo fenila ao iodo hipervalente, de modo análogo à obtenção de diariliodônios relatado por Hartmann e Meyer. ${ }^{13,48}$ Em seguida, ocorre a saída concertada de $\mathrm{TsOH}$ e $\mathrm{PhIO}_{2}$ durante a desproporção (Esquema 13).
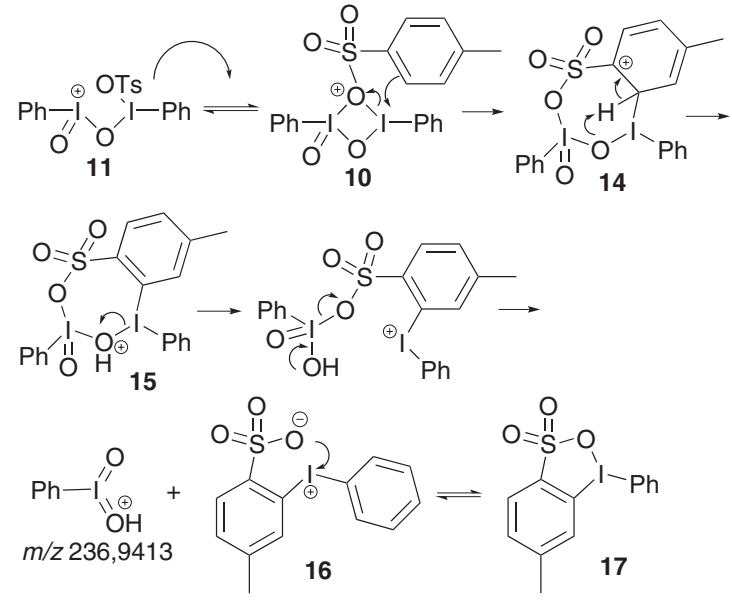

Esquema 11

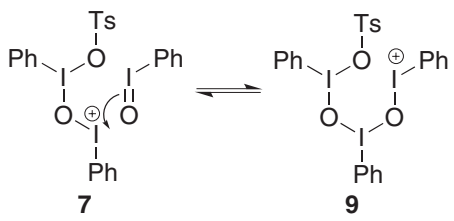

Esquema 12

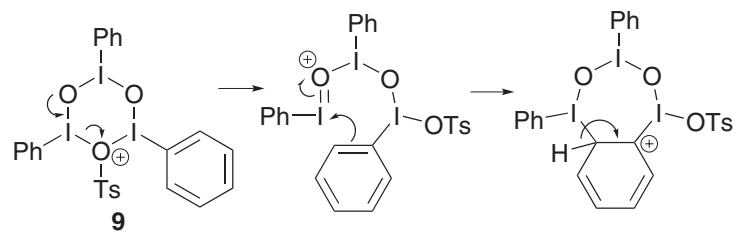

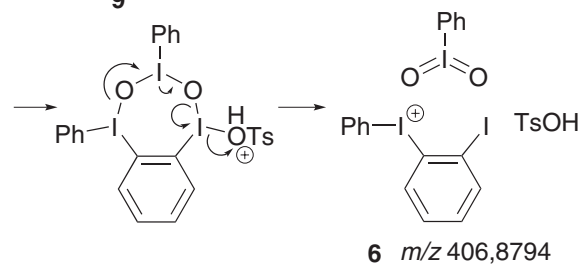

Esquema 13

O IES-EM/EM do trímero $9(\mathrm{~m} / \mathrm{z}$ 814,8243, calc. 814,8322, $-9,7 \mathrm{ppm}$ ) tem como pico base o sinal referente ao íon 6 (obs. $\mathrm{m} / \mathrm{z}$ 406,8794, calc. 406,8794, 0,0 ppm) (Figura 7). Provavelmente, a força motriz desta reação seja a saída de duas moléculas neutras, com o consequente aumento de entropia do sistema por produzir três moléculas a partir de uma (Esquema 13).

Também foram encontrados o dímero 7 (obs. $m / z$ 594,8920, calc. $594,8937,-2,9$ ppm) e o íon [PhIOTs] ${ }^{+}$(obs. $m / z, 374,9592$, calc. $374,9552,2,7$ ppm) gerados pela reação reversa de formação

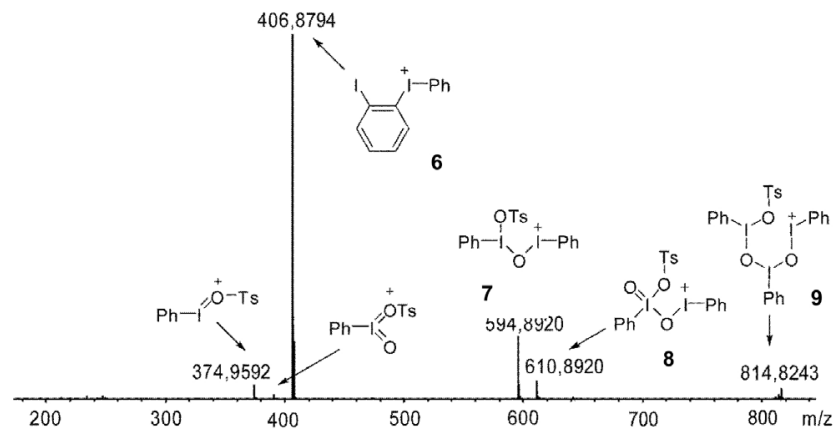

Figura 7. Espectro de IES-EM/EM do trímero 9 (energia de colisão zero) 
do trímero 9 (Esquema 12). Além disso, foi detectado o íon [PhI $(\mathrm{O})$ OTs $]^{+}(\mathrm{m} / z$ estimado 390,9501), o qual poderia ser oriundo tanto da desproporção do trímero 9 (Esquema 14), quanto pela desproporção do dímero 7 (Esquema 9).

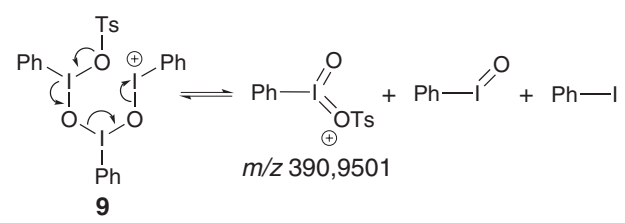

Esquema 14

\section{Proposta de mecanismo de desproporção do HTIB em acetonitrila}

Com base nas informações acima, acreditamos que o processo de desproporção do HTIB deve ocorrer de modo análogo ao DIB. ${ }^{40}$ O caminho preferencial deve ser via dímero 1 (Esquema 4). Isto ocorre porque o HTIB, devido ao seu caráter mais iônico, é capaz de formar $[\mathrm{PhIOH}]^{+}$em altas concentrações, tão logo seja dissolvido em acetonitrila. O segundo mecanismo de desproporção inicia pela reação do íon [PhIOTs] $]^{+}$com $\mathrm{PhIO}$, gerando o dímero 7, o qual desproporciona para $\mathrm{PhI}$ e $[\mathrm{PhI}(\mathrm{O}) \mathrm{OTs}]^{+}$. Este reagiria com $\mathrm{PhIO}$ produzindo o dímero $\mathbf{8}$. O equilíbrio de $\mathbf{8}$ a $\mathbf{1 1}$ permite que sejam gerados $\mathrm{PhIO}_{2}$ e o íon [PhIOTs] $]^{+}$, fechando o ciclo. Este processo representa a transformação de duas moléculas de PhIO em uma de $\mathrm{PhIO}_{2}$ e outra de PhI (Esquema 15).

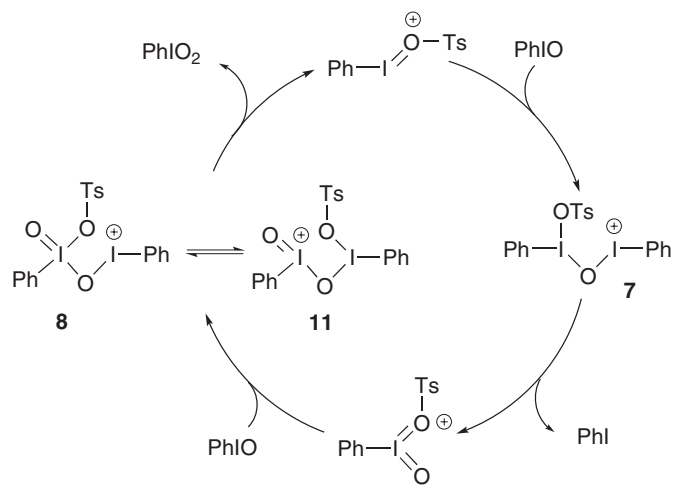

Esquema 15

A terceira rota para desproporção de HTIB parte de 9 (Esquema 16). O trímero 9 pode se decompor em: i) 8 produzindo $\mathrm{PhI}$ (Esquema 8); ii) 6 gerando $\mathrm{PhIO}_{2}$ (Esquema 13) e, iii) $[\mathrm{PhI}(\mathrm{O}) \mathrm{OTs}]^{+}, \mathrm{PhIO}$ e $\mathrm{PhI}$ (Esquema 14).

A concentração do íon [PhIOTs] $]^{+}$, que é necessário para o mecanismo do Esquema 5, é menor que a do íon $[\mathrm{PhIOH}]^{+}$(Figura 5). Este

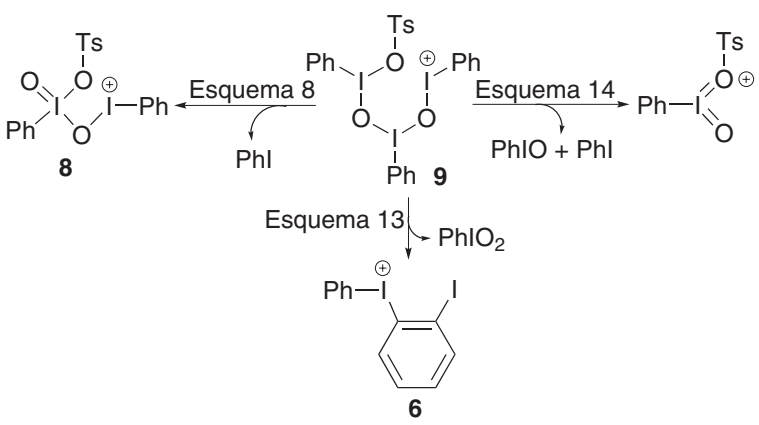

Esquema 16 dado indica que o mecanismo de desproporção do Esquema 4 deve ser o principal, enquanto que o do Esquema 16 seria secundário. Em resumo, de modo contrário à desproporção do DIB, ${ }^{40} \mathrm{o}$ HTIB parece ter preferência pela desproporção pelo caminho direto (Esquema 4) tendo a rota cíclica (Esquema 16) uma menor contribuição. Isto se deve provavelmente à maior reatividade desse reagente em relação ao DIB, porque o grupo tosila é um grupo de partida melhor que o acetila, tornando o HTIB mais eletrofílico que o DIB. Em decorrência disso, o HTIB é encontrado totalmente ionizado em solução, principalmente, na forma de $[\mathrm{PhIOH}]^{+}$.

\section{IES-EM de uma solução recém-preparada de HTIB a $80^{\circ} \mathrm{C}$}

A temperatura é um dos mais importantes fatores na cinética de reações químicas, devendo influenciar na desproporção de espécies de iodo(III). ${ }^{19,27}$ Neste sentido, Willgerodt relatou a preparação de iodilbenzeno $\left(\mathrm{PhIO}_{2}\right)$ a partir do aquecimento de iodosilbenzeno ( $\mathrm{PhIO}$ ) passando um fluxo de vapor de água, que terminava arrastando o $\mathrm{PhI}$, deixando o iodo(V) puro. ${ }^{28}$

Para investigar o efeito da temperatura no processo de desproporção, HTIB foi dissolvido em acetonitrila a $80{ }^{\circ} \mathrm{C}$ e a solução resultante foi analisada imediatamente por IES-EM de alta resolução. Na solução de HTIB recém-preparada em acetonitrila a $80{ }^{\circ} \mathrm{C}$ (Figura 8 e Tabela 1) houve o aumento da intensidade do sinal do dímero 1 em relação à mesma solução à temperatura ambiente. No entanto, as demais espécies encontradas são as mesmas presentes na solução fresca, praticamente nas mesmas proporções (Figura 3), demonstrando que a temperatura não tem um papel tão importante na desproporção do HTIB, quanto no caso do DIB. ${ }^{40}$

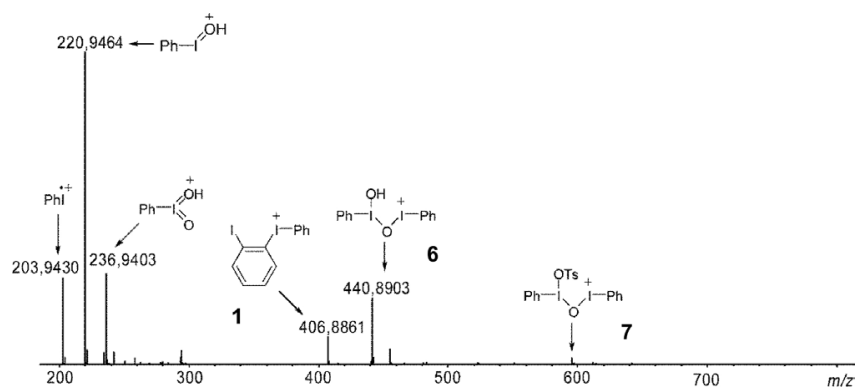

Figura 8. Espectro de massas de uma solução de HTIB em acetonitrila recém-preparada à $80^{\circ} \mathrm{C}$

Tabela 1. Espécies encontradas em solução de HTIB em acetonitrila a $80^{\circ} \mathrm{C}$

\begin{tabular}{cccc}
\hline Espécie encontrada & $m / z$ obs. & $m / z$ calc. & Erro $(\mathrm{ppm})$ \\
\hline$[\mathrm{PhI}]^{++}$ & 203,9430 & 203,9430 & 0,0 \\
{$[\mathrm{PhIOH}]^{+}$} & 220,9464 & 220,9464 & 0,0 \\
{$[\mathrm{PhI}(\mathrm{O}) \mathrm{OH}]^{+}$} & 236,9445, & 236,9413 & $-4,2$ \\
{$[(\mathrm{PhI}) \mathrm{IPh}]^{+}$} & 406,8903 & 406,8794 & 16,5 \\
{$[\mathrm{PhI}(\mathrm{OH}) \mathrm{OIPh}]^{+}$} & 440,8945 & 440,8849 & 12,2 \\
{$[\mathrm{PhI}(\mathrm{OTs}) \mathrm{OIPh}]^{+}$} & & 594,8937 & \\
\hline
\end{tabular}

A espécie 1 é formada a partir do íon $[\mathrm{PhIOH}]^{+}$. A geração de $[\mathrm{PhIOH}]^{+}$não deve necessitar de energia, pois tão logo o HTIB é dissolvido em acetonitrila ocorre sua ionização. A formação do dímero 1, principal responsável pela desproporção do HTIB, não deve ser influenciada pela temperatura. Esse contraste entre as soluções de DIB e de HTIB em acetonitrila a $80{ }^{\circ} \mathrm{C}$ leva a crer que, provavelmente, a ionização inicial do DIB seja a etapa determinante em sua desproporção, demandando energia do sistema e, portanto, sendo sensível a mudanças de temperatura. Por sua vez, o HTIB não requer 
energia para ionização devido ao melhor grupo de partida tosila, que torna o aumento da temperatura pouco relevante na desproporção.

\section{Dados de ESI-MS/MS}

A seguir, serão detalhados os principais espectros de IES-MS/MS relacionados com a discussão acima. Dados de IES-EM/EM de outras espécies mencionadas neste artigo foram discutidos anteriormente. ${ }^{40}$

O espectro de IES-EM/EM do dímero 7 (obs. $\mathrm{m} / \mathrm{z}$, 594,8976, calc. 594,8937, 6,6 ppm) (Figura 9) mostra o íon [PhI(O)OTs $]^{+}$(obs. $\mathrm{m} / \mathrm{z}$ 390,9493, calc. 390,9501, $-2,0 \mathrm{ppm}$ ) e o íon [PhIOTs] ${ }^{+}$(obs. $\mathrm{m} / \mathrm{z}$ 374,9514, calc. 374,9552, $-10,1 \mathrm{ppm}$ ) cujos mecanismos de formação foram descritos no Esquema 9. Além disso, foi detectado o cátion radical [PhOTs] ${ }^{*+}$ (obs. $\mathrm{m} / \mathrm{z}, 248,0518$, calc. 248,0507, 4,4 ppm) gerado, provavelmente, pelo ataque ipso do grupo OTs e saída de iodo radical I’ (Esquema 17).

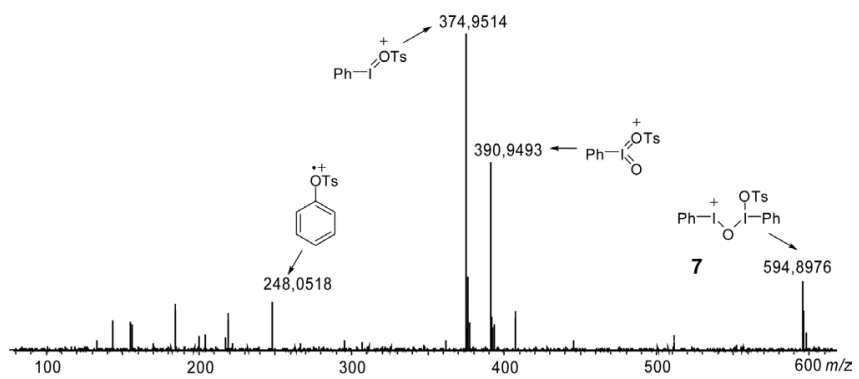

Figura 9. Espectro de IES-EM/EM do dímero 7 (energia de colisão $5 \mathrm{eV}$ )

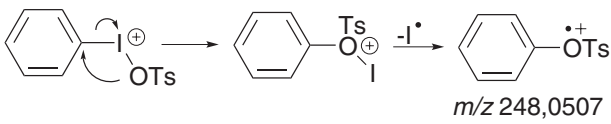

Esquema 17

O espectro de IES-EM/EM do dímero 8 (obs. $\mathrm{m} / z$ 611,3467, calc. 610,8881) (Figura 10) mostra os picos relativos ao dímero 1 (obs. $\mathrm{m} / \mathrm{z} 440,8858$, calc. 440,8849, 2,0 ppm), o íon [PhIOTs] ${ }^{+}$(obs. $\mathrm{m} / \mathrm{z}$ 374,9506 , calc. $374,9552,-12,3$ ppm) e o íon $[\mathrm{PhI}(\mathrm{O}) \mathrm{OH}]^{+}$(obs. $\mathrm{m} / z$ 236,9397, calc. 236,9413, -6,8 ppm) cujos mecanismos de formação foram discutidos anteriormente (Esquema 7). O íon $[\mathrm{PhIOH}]^{+}$(obs. $\mathrm{m} / \mathrm{z}$ 220,9466, calc. 220,9463, 1,4 ppm) deve ser gerado pela reação reversa de formação do dímero 1 (Esquema 4). Além disso, foi detectado o íon $[\mathrm{Ts}]^{+}$(obs. $\mathrm{m} / \mathrm{z}, 155,0167$, calc. 155,0167, 0,0 ppm) que deve ser proveniente da cisão heterolítica do íon [PhIOTs] $]^{+}$ou do dímero 10 (Esquema 18).

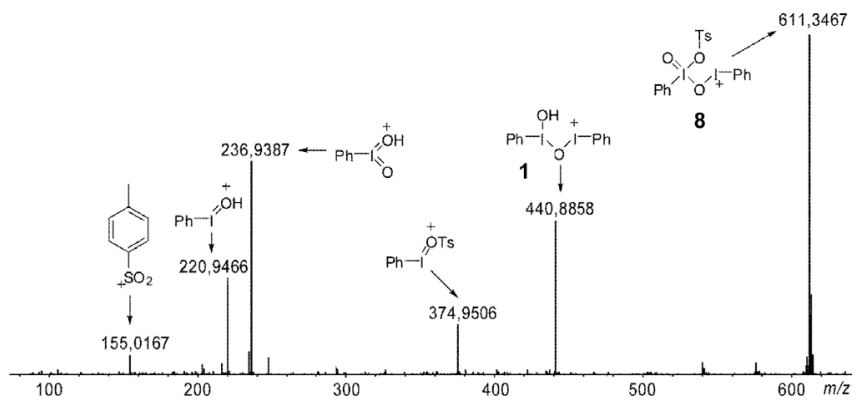

Figura 10. Espectro de IES-EM/EM do dímero 8 (energia de colisão $5 \mathrm{eV}$

O espectro de IES-EM/EM do cátion 6 (obs. $\mathrm{m} / z$ 406,8744, calc. 406,8794, -12,3 ppm) (Figura 11) revelou a presença das seguintes espécies: i) o cátion radical 2-iodobifenila (obs. $m / z, 279,9703$, calc. 279,9749, -16,4 ppm) deve ser proveniente da saída do iodo radical do di-iodeto inicial $[\mathrm{Ph}-\mathrm{PhI}]^{++}$seguido de rearranjo (Esquema 19); ii)
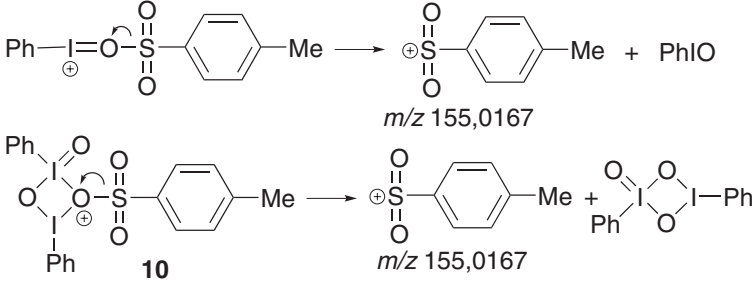

Esquema 18

o cátion do iodobenzeno (obs. $m / z, 202,9323$, calc. 202,9358, -17,2 ppm), deve ser gerado pelo ataque ipso do iodo à fenila do cátion radical 2-iodobifenila (Esquema 20); iii) o cátion bifenila $[\mathrm{Ph}-\mathrm{Ph}]^{+}$ (obs. $m / z, 153,0678$, calc. 153,0698, -13,1 ppm), é possivelmente proveniente da saída iodo radical do cátion radical 2-iodobifenila (Esquema 21) e, iv) o cátion fenila $[\mathrm{Ph}]^{+}$(obs. $\mathrm{m} / \mathrm{z}, 77,0398$, calc. $77,0391,9,1$ ppm) resultante da quebra heterolítica da ligação I(III)$\mathrm{Ph}$ do di-iodeto inicial [(PhI)IPh] ${ }^{+}$(Esquema 22).

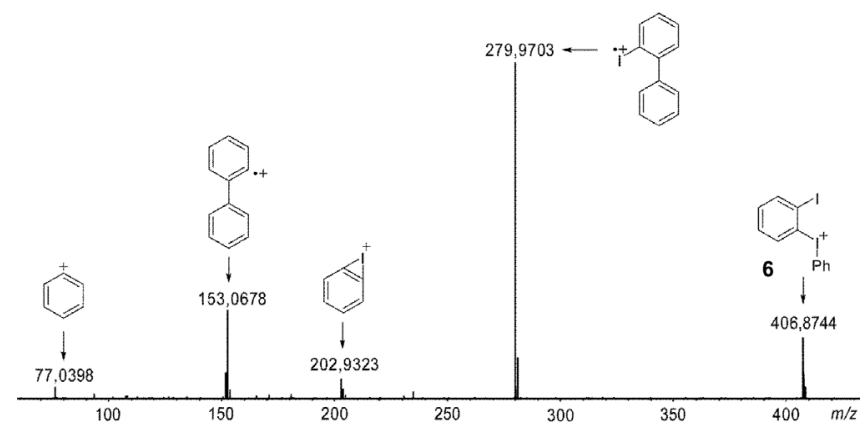

Figura 11. Espectro de IES-EM/EM do íon 6 (energia de colisão $10 \mathrm{eV}$ )

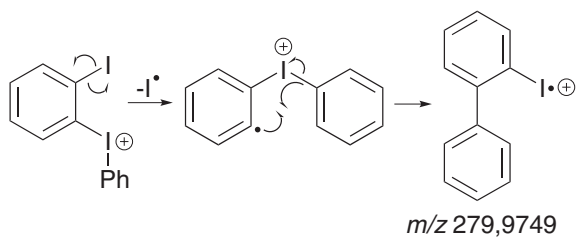

Esquema 19

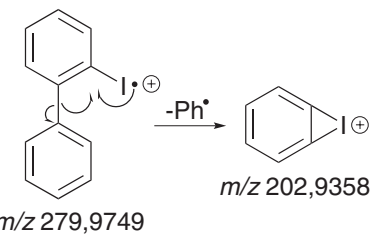

Esquema 20

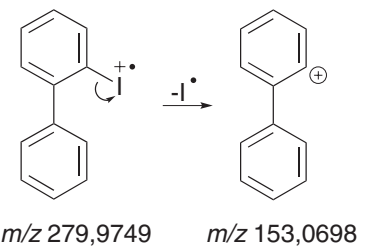

Esquema 21

No espectro de IES-EM/EM do trímero 9 (obs. $\mathrm{m} / \mathrm{z}$ 814,8260, calc. $814,8322,-7,6$ ppm) (Figura 12) verificam-se as seguintes espécies: i) o íon do dímero 8 (obs. $m / z, 610,8824$, calc. $610,8881,-9,3$ ppm) (Esquema 8); ii) o dímero 7 (obs. $m / z$ 594,8937, calc. 594,8937, 0,0 ppm) (Esquema 12); iii) o cátion 6 (obs. $\mathrm{m} / \mathrm{z}$ 406,8811, calc. 406,8794, 4,2 ppm) (Esquema 13); iv) o cátion $\left[\mathrm{PhIO}_{2} \mathrm{Ts}\right]^{+}($Esquema 


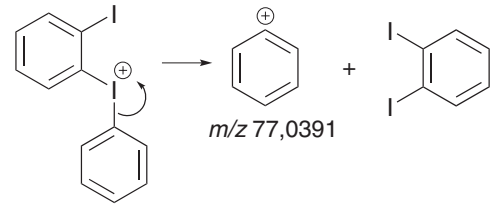

Esquema 22

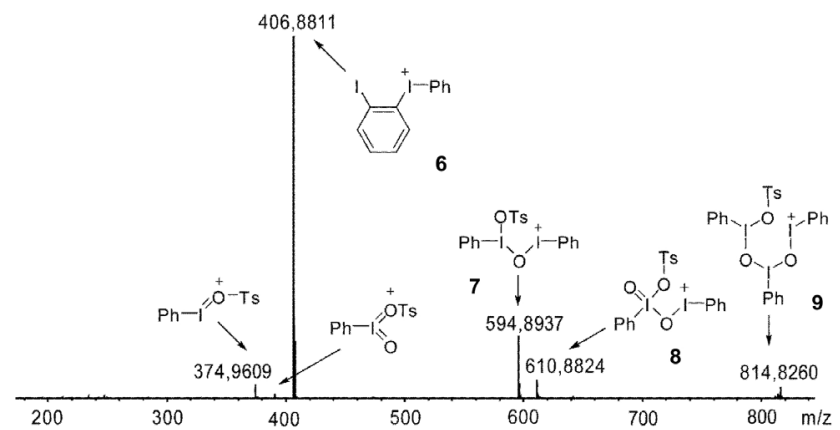

Figura 12. Espectro de IES-EM/EM do trímero 9 (energia de colisão $1 \mathrm{eV}$ )

7) e, v) o cátion [PhIOTs] $]^{+}$(obs. $m / z$ 374,9609, calc. 374,9552, 15,2 ppm) (Esquema 7).

\section{CONCLUSÕES}

O estudo por IES-EM de alta resolução da solução de HTIB em acetonitrila possibilitou entender o mecanismo de desproporção das espécies de iodo(III) em compostos de iodo(I) e iodo(V). Diferentemente do proposto para o DIB,$^{40}$ o caminho direto pelo dímero 1 deve ser o preferencial na desproporção. No mecanismo secundário cíclico estariam envolvidas espécies análogas ao DIB, como o íon [PhIOTs] $]^{+}$, os dímeros 7 e 8 e o íon de I(V) [PhI(O)OTs $]^{+}$. $\mathrm{O}$ trímero $\mathbf{9}$ também pode contribuir na desproporção formando $\mathbf{8}$, $[\mathrm{PhI}(\mathrm{O}) \mathrm{OTs}]^{+}$e 6 . A maior reatividade do HTIB provavelmente contribui para a complexidade do mecanismo no processo de desproporção. Finalmente, a reação de desproporção do HTIB não é tão influenciada pela temperatura quanto a do DIB. ${ }^{40}$

\section{AGRADECIMENTOS}

Ao contínuo apoio financeiro da FAPESP, do CNPq e da CAPES e a J. C. Tomaz pelo suporte técnico.

\section{REFERÊNCIAS E NOTAS}

1. Varvoglis, A.; Hypervalent Iodine in Organic Synthesis, Academic Press, Inc.: San Diego, 1997.

2. Wirth, T., ed.; Hypervalent iodine chemistry - Modern developments in organic synthesis, Springer: Berlin, 2003, vol. 224.

3. Moriarty, R. M.; J. Org. Chem. 2005, 70, 2893.

4. Richardson, R. D.; Wirth, T.; Angew. Chem., Int. Ed. 2006, 45, 4402.

5. Zhdankin, V. V.; Stang, P. J.; Chem. Rev. 2008, 108, 5299.

6. Pouysegu, L.; Deffieux, D.; Quideau, S.; Tetrahedron 2010, 66, 2235.

7. Zhdankin, V. V.; J. Org. Chem. 2011, 76, 1185.

8. Silva, L. F., Jr; Olofsson, B.; Nat. Prod. Rep. 2011, 28, 1722.

9. Liang, H.; Ciufolini, M. A.; Angew. Chem., Int. Ed. 2011, 50, 11849.

10. Kupper, F. C.; Feiters, M. C.; Olofsson, B.; Kaiho, T.; Yanagida, S.; Zimmermann, M. B.; Carpenter, L. J.; Luther, G. W., III,; Lu, Z.; Jonsson, M.; Kloo, L.; Angew. Chem., Int. Ed. 2011, 50, 11598.
11. Silva, L. F., Jr.; Molecules 2006, 11, 421.

12. Stang, P. J.; J. Org. Chem. 2003, 68, 2997.

13. Merritt, E. A.; Olofsson, B.; Angew. Chem., Int. Ed. 2009, 48, 9052.

14. Koser, G. F.; Aldrichimica Acta 2001, 34, 89.

15. Zhdankin, V. V.; Arkivoc 2009, 1.

16. Satam, V.; Harad, A.; Rajule, R.; Pati, H.; Tetrahedron 2010, 66, 7659.

17. Duschek, A.; Kirsch, S. F.; Angew. Chem., Int. Ed. 2011, 50, 1524.

18. Acrônimos diferentes são utilizados na literatura para os reagentes de iodo(III) mais populares. Infelizmente, não há consenso mesmo entre os especialistas na área. Os acrônimos mais empregados na literatura (DIB, PIFA e HTIB) são utilizados neste artigo.

19. Kazmierczak, P.; Skulski, L.; Kraszkiewicz, L.; Molecules 2001, 6, 881.

20. Yusubov, M. S.; Chi, K. W.; Park, J. Y.; Karimov, R.; Zhdankin, V. V.; Tetrahedron Lett. 2006, 47, 6305.

21. Zeng, X. M.; Chen, J. M.; Yoshimura, A.; Middleton, K.; Zhdankin, V. $\mathrm{V}$.; RSC Advances 2011, 1, 973.

22. Koposov, A. Y.; Karimov, R. R.; Pronin, A. A.; Skrupskaya, T.; Yusubov, M. S.; Zhdankin, V. V.; J. Org. Chem. 2006, 71, 9912.

23. Yusubov, M. S.; Zagulyaeva, A. A.; Zhdankin, V. V.; Chem. Eur. J. 2009, 15, 11091

24. Yusubov, M. S.; Nemykin, V. N.; Zhdankin, V. V.; Tetrahedron 2010, 66, 5745.

25. Tohma, H.; Takizawa, S.; Maegawa, T.; Kita, Y.; Angew. Chem., Int. Ed. 2000, 39, 1306.

26. Tohma, H.; Maegawa, T.; Takizawa, S.; Kita, Y.; Adv. Synth. Catal. 2002, $344,328$.

27. Richter, H. W.; Cherry, B. R.; Zook, T. D.; Koser, G. F.; J. Am. Chem. Soc. 1997, 119, 9614.

28. Willgerodt, C.; Ber. 1892, 25, 3494.

29. Silva, L. F., Jr.; Lopes, N. P.; Tetrahedron Lett. 2005, 46, 6023.

30. Zhdankin, V. V.; Koposov, A. E.; Smart, J. T.; Tykwinski, R. R.; McDonald, R.; Morales-Izquierdo, A.; J. Am. Chem. Soc. 2001, 123, 4095.

31. Fabris, D.; Mass Spectrom. Rev. 2005, 24, 30.

32. Santos, L. S.; Knaack, L.; Metzger, J. O.; Int. J. Mass Spectrom. 2005, 246,84 .

33. Santos, L. S.; J. Braz. Chem. Soc. 2011, 22, 1827.

34. Paz-Schmidt, R. A.; Bonrath, W.; Plattner, D. A.; Anal. Chem. 2009, 81, 3665.

35. Coelho, F.; Eberlin, M. N.; Angew. Chem., Int. Ed. 2011, 50, 5261.

36. Sam, J. W.; Tang, X. J.; Magliozzo, R. S.; Peisach, J.; J. Am. Chem. Soc. 1995, 117, 1012.

37. Li, Z.; Tang, Z. H.; Hu, X. X.; Xia, C. G.; Chem. Eur. J. 2005, 11, 1210.

38. Ochiai, M.; Miyamoto, K.; Suefuji, T.; Shiro, M.; Sakamoto, S.; Yamaguchi, K.; Tetrahedron 2003, 59, 10153.

39. Yu, J.; Tian, J.; Zhang, C.; Adv. Synth. Catal. 2010, 352, 531.

40. Silva, L. F., Jr.; Vasconcelos, R. S.; Lopes, N. P.; Int. J. Mass Spectrom. 2008, 276, 24

41. Merritt, E. A.; Carneiro, V. M. T.; Silva, L. F., Jr; Olofsson, B.; J. Org. Chem. 2010, 75, 7416.

42. Vessecchi, R.; Crotti, A. E. M.; Guaratini, T.; Colepicolo, P.; Galembeck, S. E.; Lopes, N. P.; Mini Rev. Org. Chem. 2007, 4, 75.

43. Masson, I.; Race, E.; Pounder, F. E.; J. Chem. Soc. 1935, 1669.

44. Togo, H.; Aoki, M.; Yokoyama, M.; Tetrahedron Lett. 1991, 32, 6559.

45. Opitz, G.; Angew. Chem., Int. Ed. 1967, 6, 107.

46. King, J. F.; Acc. Chem. Res. 1975, 8, 10.

47. Cremlyn, R. J.; Saunders, D.; Phosphorus, Sulfur Silicon Relat. Elem. 1993, 81, 73.

48. Hartmann, C.; Meyer, V.; Chem. Ber. 1894, 27, 426. 